\title{
Development of a Novel and Simple Method to Evaluate Disintegration of Rapidly Disintegrating Tablets
}

\author{
Yohei Hoashi, ${ }^{a, b}$ Yuichi Tozuka, ${ }^{b, \dagger}$ and Hirofumi Takeuchi*,b \\ ${ }^{a}$ Pharmaceutical Research Center, Nipro Corporation; 3023 Nojicho, Kusatsu, Shiga 525-0055, Japan: and \\ ${ }^{b}$ Laboratory of Pharmaceutical Engineering, Gifu Pharmaceutical University; 1-25-4 Daigaku Nishi, Gifu 501-1196, \\ Japan. \\ Received June 5, 2013; accepted June 10, 2013
}

\begin{abstract}
The purpose of this study was to develop and test a novel and simple method for evaluating the disintegration time of rapidly disintegrating tablets (RDTs) in vitro, since the conventional disintegration test described in the pharmacopoeia produces poor results due to the difference of its environmental conditions from those of an actual oral cavity. Six RDTs prepared in our laboratory and 5 types of commercial RDTs were used as model formulations. Using our original apparatus, a good correlation was observed between in vivo and in vitro disintegration times by adjusting the height from which the solution was dropped to $8 \mathrm{~cm}$ and the weight of the load to $10 \mathrm{or} 20 \mathrm{~g}$. Properties of RDTs, such as the pattern of their disintegrating process, can be assessed by verifying the load. These findings confirmed that our proposed method for an in vitro disintegration test apparatus is an excellent one for estimating disintegration time and the disintegration profile of RDTs.
\end{abstract}

Key words rapidly disintegrating tablets; disintegration test; disintegration time; disintegrating profile

In recent years, several types of dosage forms have been developed for a variety of demands such as improvement in patient compliance. Among them, rapidly disintegrating tablets (RDTs) are a convenient dosage form for elderly people or children who have difficulty swallowing conventional tablets or capsules, as RDTs are disintegrated within several tens of seconds with a small amount of saliva in the oral cavity. ${ }^{1-5)}$ RDTs are also applicable for drug administration through an enteral feeding tube without crushing the tablets, since RDTs are easily dispersible into an aqueous solution.

Two main factors are important for disintegration of RDTs in the oral cavity: the absorption of saliva and the destruction by external pressure between the tongue and the upper palate. Evaluating the disintegration profile of RDTs is important in their development and quality assurance. Many studies have recorded disintegration times in the human mouth. ${ }^{6,7)}$ However, evaluating the disintegration time in the human mouth entails the following problems: 1) the disintegration time in the mouth tends to depend on oral environments and conditions, and thus, results are scattered; and 2) the human sensory test has risks for healthy volunteers. Many other disintegration test methods have been proposed such as a modified disintegration test and a modified dissolution test. ${ }^{89}$ ) However, the evaluation of various RDTs using these methods is ultimately flawed because their environments differ from that of the oral cavity. Some researchers reported disintegration test for RDTs using a loading apparatus. ${ }^{10-12)}$ These apparatuses still have some problems, such as wetting of the tablet only on one side and the requirement of a special apparatus such as a texture analyzer to load.

The purpose of this study was to develop a novel and simple method to simulate the disintegration time and profiles of RDTs in the oral cavity. The developed method has several advantages: 1) it involves a simple apparatus that can be easily

The authors declare no conflict of interest.

† Present address: Osaka University of Pharmaceutical Sciences; 4-20-1 Nasahara, Takatsuki, Osaka 569-1094, Japan. constructed in the laboratory; 2) the environment is adjustable as for the oral cavity; and 3) two aspects of the tablet disintegration can be evaluated. In this study, we used 5 commercial RDTs as well as 6 RDTs prepared by our own methods as model RDTs, and evaluated the correlation between in vivo and in vitro disintegration times. The differences in the disintegration profile among RDTs, with regard to the additives used, were assessed by verifying the apparatus conditions.

\section{Experimental}

Materials Mannitol (ROQUETTE Japan K.K., Japan) and erythritol (Mitsubishi-Kagaku Foods Co., Japan) were used as the excipient of the RDTs. Composite particles made of porous silica (Sylysia 350, Fuji Silysia Chemical Ltd., Japan) were used, with mannitol and erythritol as a binder. Two grades of crospovidone (Kollidon CL, Kollidon CL-M, BASF Japan Ltd., Japan), croscarmellose sodium (Ac-Di-Sol, Dainippon Sumitomo Pharma Co., Ltd., Japan) and low-substituted hydroxypropyl cellulose (L-HPC32, Shin-Etsu Chemical Co., Ltd., Japan) were used as the disintegrant. Magnesium stearate (Mg-st, Taihei Chemical Industrial Co., Ltd., Japan) was used as the lubricant. Takepron OD (Takeda Pharmaceutical Co., Ltd., Japan), Triazolam EMEC, Enalapril EMEC (Elmed Eisai Co., Ltd., Japan), Gaster D (Astellas Pharma Inc., Japan) and Lendormin D (Nippon Boehringer Ingelheim Co., Ltd., Japan), were selected as representative commercial RDTs. The commercial RDTs were named "tablet A" to "tablet E," respectively. All other reagents were of analytical grade.

Preparation of RDTs Test RDTs were prepared according to a patented method by Takeuchi et al., in which composite particles of porous silica are used with sugar alcohol as a binder. ${ }^{13)}$ The composite particles were prepared in the following method. One kilogram of porous silica was suspended in $100 \mathrm{~L}$ of solution composed of $1.5 \%$ mannitol or $2 \%$ erythritol, and the suspension was fed into a spray dryer (NB-9.7, Ohkawara Kakohki Co., Ltd., Japan). The spray-dried products were defined as composite particles of mannitol and silica (MSCP) or erythritol and silica (ESCP). Tablets formulations 
Table 1. Formulation and Properties of Test RDTs

\begin{tabular}{|c|c|c|c|c|c|c|}
\hline Formulation & 1 & 2 & 3 & 4 & 5 & 6 \\
\hline Mannitol & 148 & & 148 & 158 & 178 & \\
\hline Erythritol & & 148 & & & & 178 \\
\hline MSCP & 30 & & 30 & 20 & 10 & \\
\hline ESCP & & 30 & & & & 10 \\
\hline Kollidon CL & 20 & 20 & & & & \\
\hline Ac-di-sol & & & 20 & & & \\
\hline L-HPC & & & & 20 & & \\
\hline Kollidon CL-M & & & & & 10 & 10 \\
\hline Mg-st & 2 & 2 & 2 & 2 & 2 & 2 \\
\hline Total (mg) & 200 & 200 & 200 & 200 & 200 & 200 \\
\hline Compression (MPa) & 150 & 150 & 150 & 150 & 150 & 150 \\
\hline Diameter (mm) & 8 & 8 & 8 & 8 & 8 & 8 \\
\hline Hardness (N) & $75.7 \pm 9.8$ & $87.7 \pm 3.8$ & $64.7 \pm 1.5$ & $88.7 \pm 13.3$ & $66.7 \pm 2.1$ & $81.7 \pm 2.5$ \\
\hline In vivo disintegration time (s) & $21.5 \pm 2.4$ & $19.3 \pm 2.5$ & $47.0 \pm 5.7$ & $41.7 \pm 3.7$ & $16.0 \pm 2.1$ & $16.0 \pm 2.6$ \\
\hline
\end{tabular}

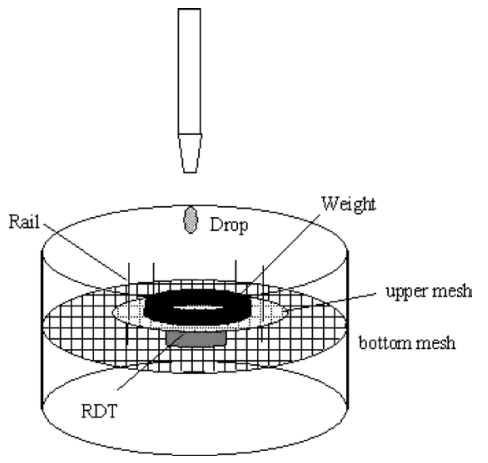

Fig. 1. Schematic Representation of the New Apparatus for Disintegration Test of RDTs

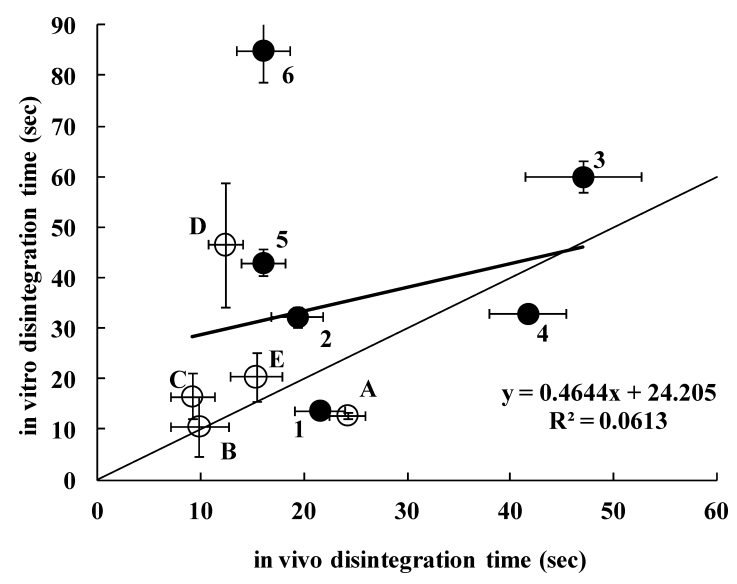

Fig. 2. Disintegration Time of RDTs as Measured by the Human Sensory Test vs. That by the Conventional Disintegration Test

Closeted circles indicate RDTs prepared in our laboratory; open circles, commercial RDTs. Values are the mean \pm S.D.; thick line, regression line; thin line, $y=x$ line.

are listed in Table 1. The formulations were directly compressed into tablets by a hydraulic press machine (N3043-00, Riken Seiki Co., Ltd., Japan) equipped with flat-faced punches $8 \mathrm{~mm}$ in diameter.

Human Sensory Test Disintegration time in the oral cavity was assessed by 6 healthy volunteers. Prior to the test, all
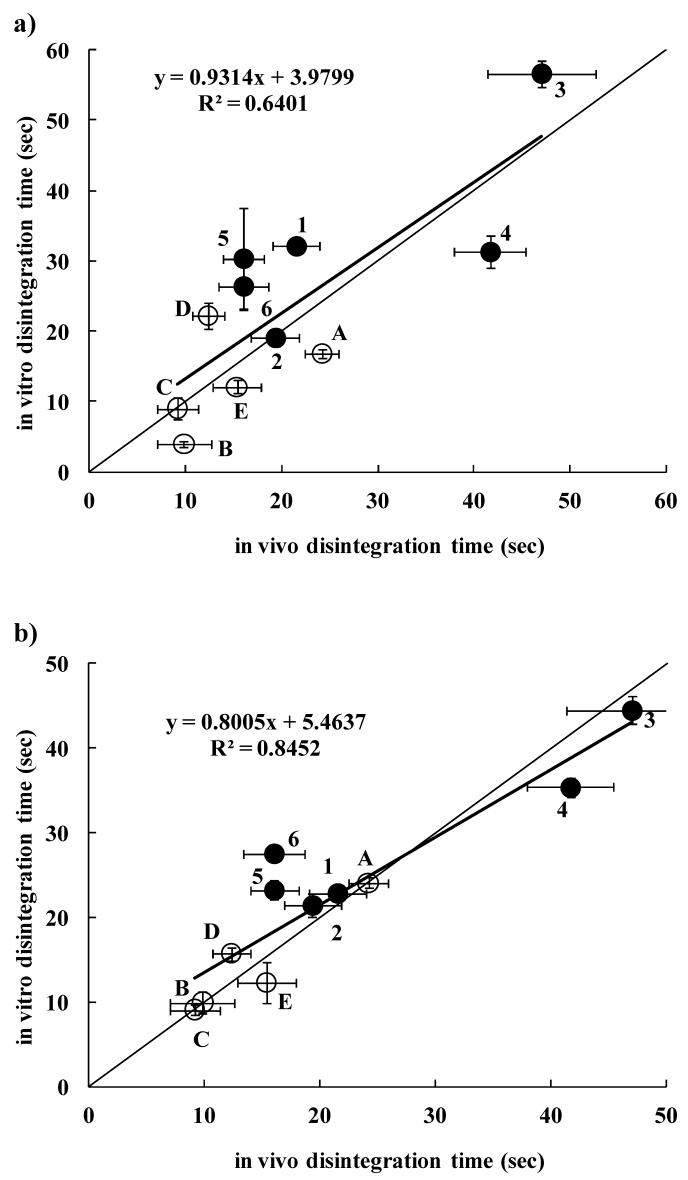

Fig. 3. Correlation Plots of in Vivo/in Vitro Disintegration Times Using the New Disintegration Test with (a) Water or (b) an Artificial Saliva Solution

Closeted circles indicate RDTs prepared in our laboratory; open circles, commercial RDTs. Values are the mean \pm S.D.; thick line, regression line; thin line, $y=x$ line.

volunteers were briefed in detail on the purpose of the test and gave informed consent. A tablet was placed on the tongue, and subjects were allowed to move the tablet against the upper palate of the mouth with their tongue. Disintegration time was 
a)

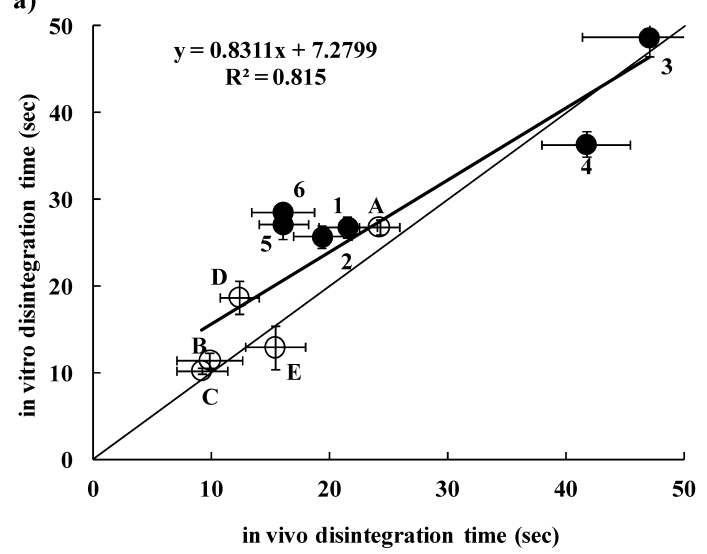

c)

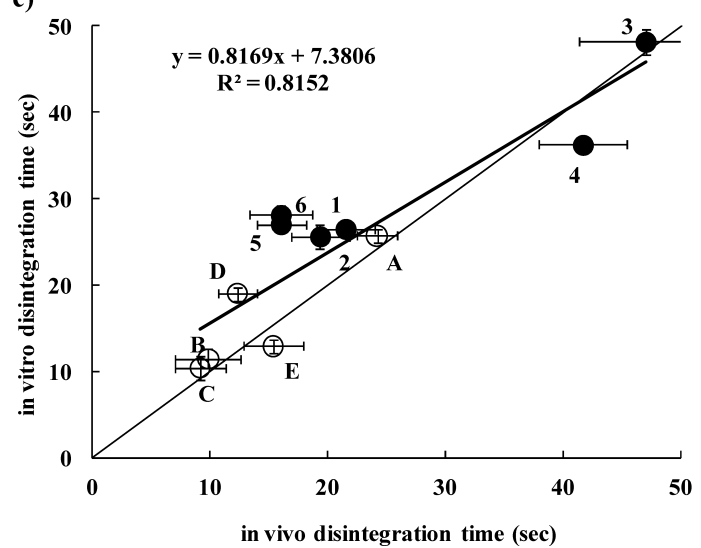

b)

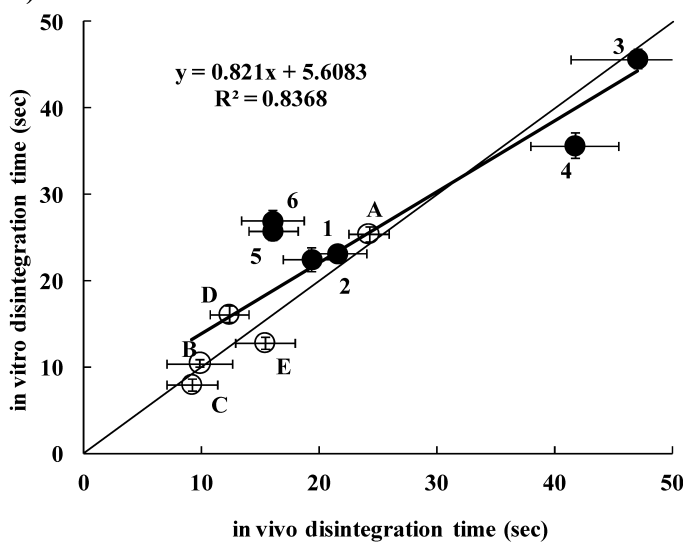

d)

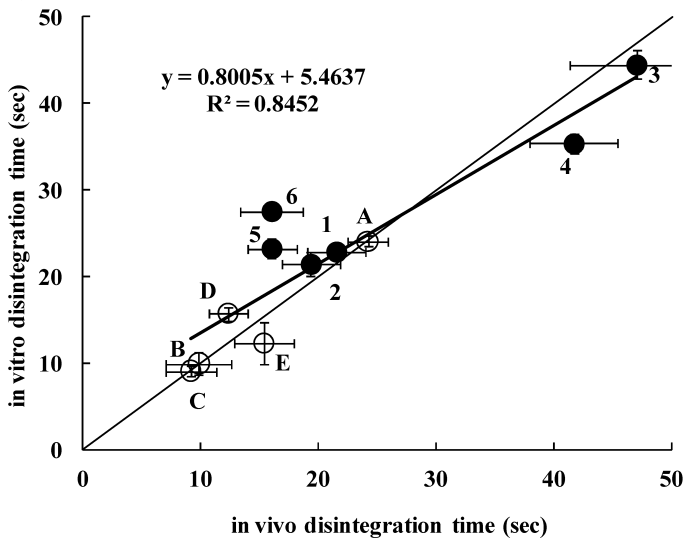

Fig. 4. Effect of Flow Rate and Height Dropped on Correlation of in Vivo/in Vitro Disintegration Times in the New Disintegration Test

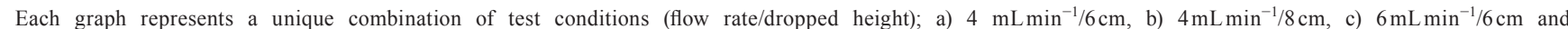
d) $6 \mathrm{~mL} \mathrm{~min}-1 / 8 \mathrm{~cm}$. Closeted circles indicate RDTs prepared in our laboratory; open circles, commercial RDTs. Values are the mean \pm S.D.; thick line, regression line; thin line, $y=x$ line.

recorded when the subject felt that the tablet had disintegrated completely in his mouth. Disintegrated powder was immediately spat out and rinsed out of the subject's mouth with water. The mean oral disintegration time represents the average and the standard deviation. This study was conducted according to the protocol approved and reviewed by the Institutional Ethics Committee.

Conventional Disintegration Test Disintegration time of RDTs was also determined following the method described in the Japanese Pharmacopoeia XVI without an auxiliary disk. All measurements were performed three times, and the mean disintegration time represents the average and the standard deviation.

New in Vitro Disintegration Test for RDTs Figure 1 shows our proposed disintegration test apparatus, composed of two meshes, a flow pump, a ring-shaped weight and an incubator. A tablet was put on the mesh and covered with another mesh fastened with slide rails, with or without a 10,20 or $40 \mathrm{~g}$ ring-shaped weight on the upper mesh as a load. The disintegration test solution, which was kept at $37^{\circ} \mathrm{C}$ in an incubator, was dropped above the tablet with a flow pump; the inner diameter of the top of the tube was $3.5 \mathrm{~mm}$. The disintegration test solution was distilled water or artificial saliva solution composed $1.44 \mathrm{~g} / \mathrm{L} \mathrm{NaCl}, 1.47 \mathrm{~g} / \mathrm{L} \mathrm{KCl}$ and $0.3 \%$ polysorbate 80. The flow rate and height dropped was set at 4 or $6 \mathrm{~mL} / \mathrm{min}$ and 6 or $8 \mathrm{~cm}$, respectively. Disintegration time was recorded when disintegration of RDT was complete, and completion of disintegration was determined by the upper mesh contacting the bottom mesh. All measurements were performed three times, and the mean disintegration time represents the average and the standard deviation.

\section{Results and Discussion}

Conventional Disintegration Test Figure 2 shows the correlation plots of the disintegration time between the human sensory test and the conventional disintegration test specified in the Japanese pharmacopoeia. Disintegration time of tablets 2, 3, 5, 6 and D was slow compared to that in vivo, in spite of a much large amount of water than that in the human oral cavity. It was indicated that no external pressure was applied in the conventional in vitro test. Thus, a good correlation was not observed between the human sensory test and the conventional disintegration test. These results confirmed that the conventional disintegration test was not sufficient to evaluate disintegration of RDTs.

Determination of Disintegration Test Solution in the New Disintegration Test A new apparatus by considering the defects of the conventional disintegration test such as the amount of medium and lack of external pressure was constructed as shown in Fig. 1. Process variables for our new apparatus are types of solution, magnitude of impact from the dropped solution, flow rate of the solution, and force loaded 
on the tablet. We tried to adjust the conditions of this method to those of the oral cavity by altering these four parameters.

Figure 3 shows the relationship between disintegration time in the oral cavity and our newly designed disintegration test, where distilled water or artificial saliva solution was used as disintegration test solutions. The test conditions were kept at $6 \mathrm{~mL} / \mathrm{min}$ of flow rate and $8 \mathrm{~cm}$ of drop height for both solutions. The disintegration test with our apparatus obviously shows a higher correlation than that of the conventional disintegration test. The squared $R$ value for artificial saliva solution is slightly higher than that for distilled water, suggesting that the correlation between the in vivo and in vitro study was improved by using artificial saliva solution. When the saliva solution was used as test solution, in contrast to the test with distilled water, disintegration times of tablets $3, \mathrm{~A}$ and $\mathrm{D}$ were similar to those measured in vivo. Tablet 3 and A are disintegrated by absorption of water into the disintegrant. Tablet $\mathrm{D}$ having porous structure is disintegrated by absorption of water by capillary action into pore structure. The wetting, absorption and capillary property of RDTs was affected by the surface activity and the osmotic pressure of the test solution. The artificial saliva solution was more suitable for measuring the disintegration time with the new method than distilled water.

Determination of the Test Condition in the New Disintegration Test The effect of flow rate and height dropped of the test solution on the in vivo/in vitro correlation is shown in Fig. 4. The disintegration times of each tablet were almost the same for flow rates of 4 and $6 \mathrm{~mL} / \mathrm{min}$. This result indicated that disintegration time was not affected by water volume. On the other hand, higher correlation between in vivo and in vitro disintegration time was observed with the dropped height of $8 \mathrm{~cm}$ than that of $6 \mathrm{~cm}$. This result suggested that disintegration of some tablet were accelerated by the impact of dropped the test solution. The in vitro disintegration times of tablets 5 and 6 were significantly slower than those in the human sensory test, which might be due to formation of gel-like structure with high viscosity in the tablet during the disintegration process, since these tablets were formulated with micronized crospovidone (Kollidon CL-M). Although the effect of the height of the test solution on the correlation difference among those samples is small, the higher dropped height produced a better result. This is due to the difference in the impact of falling; however, the correlation could not be improved drastically at heights greater than 8 .

A ring-shaped weight was set on the upper mesh to represent the crushing force between the tongue and the upper palate in the mouth. Figure 5 shows the result of the new disintegration test with 10,20 and $40 \mathrm{~g}$ load under $6 \mathrm{~mL} / \mathrm{min}$ of flow rate and $8 \mathrm{~cm}$ of dropped height. The correlation between the in vivo and in vitro test increased with the weight loaded on the tablet. The disintegration times of tablets 5 and 6 , in contrast to the test without a load, exhibited good correlation. This result indicated that these tablets gradually destruct by absorbing water due to their sticky state. By optimizing the process variables in our new disintegration apparatus, a good relationship between the in vitro and in vivo tests was observed. The relatively low value of squared $R$ under optimum conditions was attributed to the large deviation of the human sensory test owing to individual differences.

Consideration on Disintegrating Profile of RDTs Figure
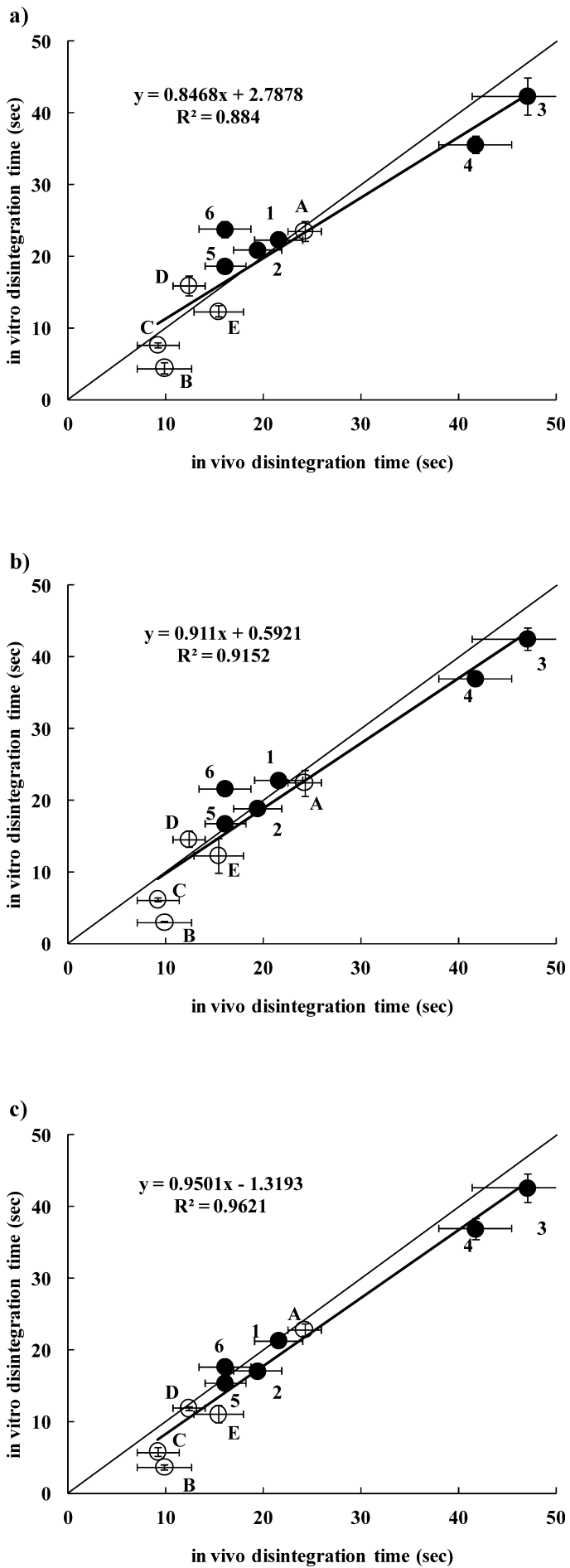

Fig. 5. Correlation Plots of in Vivo/in Vitro Disintegration Times Using the New Disintegration Test with a) $10 \mathrm{~g}$, b) $20 \mathrm{~g}$ and c) $40 \mathrm{~g}$ of Weight on the Tablet

Closeted circles indicate RDTs prepared in our laboratory; open circles, commercial RDTs. Values are mean \pm S.D.; thick line, regression line; thin line, $y=x$ line.

6 shows the effect of loading weight on the disintegration time of test tablets. Disintegration times of tablets 1, 3, 4, A and E were not affected by changing the weight. These tablets have common features with respect to additives used, manufacturing methods and tablet structure. Tablets 1, 3 and 4 were formulated with mannitol as an excipient and with crospovidone, croscarmellose sodium and L-HPC as a disintegrants. 


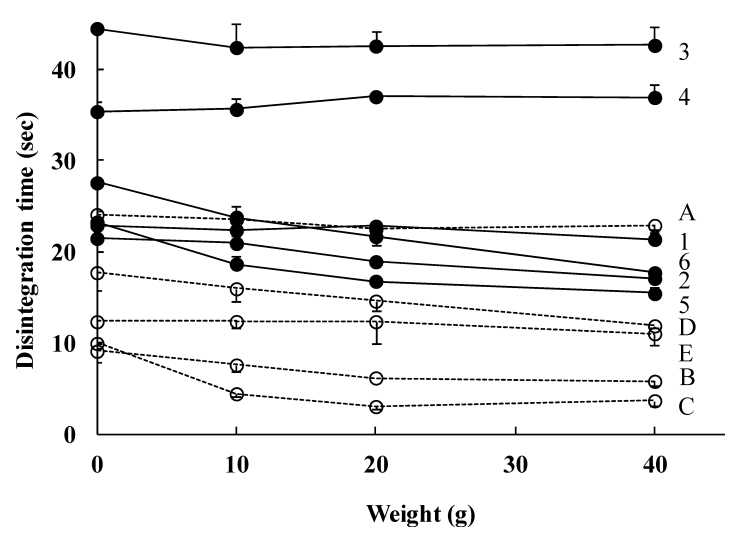

Fig. 6. Changes in Disintegration Times of RDTs by Weight Values are the mean \pm S.D.

In these formulations, the viscosity of the dissolved additives with absorbed water is low in the tablets, leading to the rapid destruction of the tablet. In addition, tablets 1, 3 and 4 have a dense structure because they are prepared by direct compression. Tablets A and E are also formulated with low-stickiness additives during water absorption, and have a dense structure from their manufacture by direct compression. ${ }^{14,15)}$ On the other hand, the disintegration time of the other tablets $(2,5,6$, B, C, D) decreased with an increase in weight. Tablets 2 and 6 contain erythritol, and tablets 5 and 6 contain micronized crospovidone. Since erythritol and micronized crospovidone demonstrate a sticky state by water absorption, the destructing process of tablets 2, 5 and 6 was slow. By contrast, the porous structure of tablets $\mathrm{B}, \mathrm{C}$ and $\mathrm{D}$ became fragile by absorbing water. $^{16,17)}$ Therefore, the destructing process of these tablets was particularly susceptible to weight.

The disintegration process of RDTs proceeds through two steps: water permeation into the tablet, and the destruction followed by reduction of the binding force between particles. Using our disintegration apparatus, it is possible to classify the disintegrating mechanism of RDTs in terms of which step controls the disintegration of RDTs. When the load is put on the tablet, a restitutive force against the load is generated in the tablet. This force decreases with water absorption; thus, the tablet structure begins to break when the repulsion power becomes smaller than the load. The disintegration process of tablets that are not affected by weight, such as tablets 1 , 3, 4, A and E, would be controlled by the water absorption step, since these tablets break promptly after some moisture is absorbed. In the case of tablets 2, 5, 6, B, C and D, in which the disintegration process was affected by weight, the disintegration time is determined by the destructing step, since the tablet structure breaks slowly by water absorption. It was concluded that the disintegration mechanism could be classified into two patterns according to whether the disintegration time of RDTs was influenced by the loading weight.

\section{Conclusion}

We developed a novel and simple disintegration test apparatus for evaluating the disintegration time of RDTs. This apparatus, composed of a simple device, composes a similar environment to that of the oral cavity. Therefore, the disintegration time of all tablets tested corresponded to that in oral cavity. An excellent correlation was found between their in vivo and in vitro disintegration times. Recomended test condition was set at $6 \mathrm{~mL} / \mathrm{min}$ of flow rate, $8 \mathrm{~cm}$ of drop height and $20-40 \mathrm{~g}$ loaded since high correlation was found between in vivo and in vitro disintegration time under these conditions. Using this method, the disintegration process of RDTs could be classified in terms of whether its disintegration time is affected by load.

\section{References}

1) Seager H., J. Pharm. Pharmacol., 50, 375-382 (1998).

2) Shu T., Suzuki H., Hironaka K., Ito K., Chem. Pharm. Bull., 50, 193-198 (2002).

3) Kuno Y., Kojima M., Ando S., Nakagami H., J. Controlled Release, 105, 16-22 (2005).

4) Sugimoto M., Maejima T., Narisawa S., Matsubara K., Yoshino H., Int. J. Pharm., 296, 64-72 (2005).

5) Sugimoto M., Narisawa S., Matsubara K., Yoshino H., Nakano M., Handa T., Int. J. Pharm., 320, 71-78 (2006).

6) Sugimoto M., Matsubara K., Koida Y., Kobayashi M., Pharm. Dev. Technol., 6, 487-493 (2001).

7) Fukami J., Ozawa A., Yoshihashi Y., Yonemochi E., Terada K., Chem. Pharm. Bull., 53, 1536-1539 (2005).

8) Watanabe Y., Koizumi K., Zama Y., Kiriyama M., Matsumoto Y., Matsumoto M., Biol. Pharm. Bull., 18, 1308-1310 (1995).

9) Bi Y. X., Sunada H., Yonezawa Y., Danjo K., Drug Dev. Ind. Pharm., 25, 571-581 (1999).

10) Abdelbary G., Eouani C., Prinderre P., Joachim J., Reynier J., Piccerelle P., Int. J. Pharm., 292, 29-41 (2005).

11) Dor P. J., Fix J. A., Pharm. Dev. Technol., 5, 575-577 (2000).

12) Narazaki R., Harada T., Takami N., Kato Y., Ohwaki T., Chem. Pharm. Bull., 52, 704-707 (2004).

13) Takeuchi H., Tanimura S., JP Patent 248922A (2006).

14) Shimizu T., Sugaya M., Nakano Y., Izutsu D., Mizukami Y., Okochi K., Tabata T., Hamaguchi N., Igari Y., Chem. Pharm. Bull., 51, 1121-1127 (2003).

15) Wada K., Kurosaki S., Oki S., JP Patent 162613A (2005).

16) Mizumoto T., Masuda Y., Yamamoto T., Yonemochi E., Terada K., Int. J. Pharm., 306, 83-90 (2005).

17) Morita Y., Tsushima Y., Yasui M., Termoz R., Ajioka J., Takayama K., Chem. Pharm. Bull., 50, 1181-1186 (2002). 